Original Article

\title{
The Renal Resistive Index in systemic sclerosis: Determinants, prognostic implication and proposal for specific age-adjusted cut-offs
}

\author{
Cosimo Bruni ${ }^{\mathrm{a}, \mathrm{i}, *}$, Edoardo Rosato $^{\mathrm{b}}$, Vanessa Maestripieri ${ }^{\mathrm{c}}$, Antonietta Gigante ${ }^{\mathrm{b}}$, Giulia Tesei $^{\mathrm{a}}$, \\ Silvia Bellando-Randone ${ }^{\mathrm{a}, \mathrm{d}}$, Serena Guiducci ${ }^{\mathrm{a}, \mathrm{d}}$, Marco Chiostri ${ }^{\mathrm{b}}$, Khadija El Aoufy ${ }^{\mathrm{a}}$, \\ Jelena Blagojevic $^{\mathrm{a}, \mathrm{d}}$, Alberto Moggi-Pignone ${ }^{\mathrm{b}, \mathrm{e}}$, Amato De Paulis ${ }^{\mathrm{f}}$, Daniel E. Furst ${ }^{\mathrm{a}, \mathrm{d}, \mathrm{g}, \mathrm{h}}$, \\ Maria Boddi ${ }^{\mathrm{b}, \mathrm{h}, 1}$, Marco Matucci-Cerinic ${ }^{\mathrm{a}, \mathrm{d}, 1}$ \\ ${ }^{a}$ Department of Experimental and Clinical Medicine, Division of Rheumatology, University of Florence, Via Delle Oblate 4, 50134 Florence, Italy \\ ${ }^{\mathrm{b}}$ Department of Translational and Precision Medicine, Sapienza University of Rome, Rome, Italy \\ ${ }^{\mathrm{c}}$ Department of Experimental and Clinical Medicine, University of Florence, Florence, Italy \\ ${ }^{\mathrm{d}}$ Department of Geriatric Medicine, Division of Rheumatology, Azienda Ospedaliera Universitaria Careggi, Florence, Italy \\ ${ }^{\mathrm{e}}$ Department of Internal Medicine, Division of Internal Medicine Unit III, Azienda Ospedaliera Universitaria Careggi, Florence, Italy \\ ${ }_{\mathrm{f}}^{\mathrm{f}}$ Department of Translational Medical Sciences, Center for Basic and Clinical Immunology Research (CISI), WAO Center of Excellence, University Federico II, Naples, Italy \\ ${ }^{\mathrm{g}}$ Department of Medicine, Division of Rheumatology, University of California at Los Angeles, USA \\ ${ }^{\mathrm{h}}$ University of Washington, Seattle, WA, USA \\ ${ }^{\mathrm{i}}$ Department Cardio-Thorax-Vascular Medicine, Division of General Cardiology, Azienda Ospedaliera Universitaria Careggi, Florence, Italy
}

\section{A R T I C L E I N F O}

\section{Keywords:}

Renal artery Doppler ultrasound

Renal Resistive Index

Vasculopathy

Fibrosis

Prognosis

Systemic sclerosis

\begin{abstract}
A B S T R A C T
Background: Renal Resistive Index (RRI), reflects changes in both renal vascular and tubular-interstitial compartments and in systemic vascular compliance related to age and comorbidities.

Objectives: a) To investigate determinants of RRI in SSc population, b) its association with SSc-related features and c) to test its prognostic impact on organ specific worsening or death.

Methods: 380 SSc patients $\geq 18$ years were enrolled after giving informed consent. Baseline data on RRI, laboratory, instrumental and therapeutic features were retrospectively collected. Age-SSc adjusted cut-offs were created by dividing the population in age quartiles and considering RRI values $>75$ th percentile as pathologic. Clinical follow-up was performed until last available visit or the development/worsening of specific internal organ involvement or death.

Results: RRI was independently predicted by age and systolic pulmonary arterial pressure on Echo. Therefore, we created Age-SSc adjusted pathologic RRI cut-offs, which were significantly associated with various disease related skin and lung fibrotic manifestations, as well as vasculopathic complications. After a mean follow-up of $3.6 \pm 2.6$ years, RRI was one of the independent predictors (together with modified Rodnan skin score, interstitial lung disease, presence of dyspnoea and late nailfold-videocapillaroscopy pattern) for mortality, with 0.68 as best cut-off (sensitivity $88.5 \%$, specificity $50.9 \%$ ).

Conclusion: If corroborated, Renal Resistive Index cut-offs might be used to evaluate renal and extrarenal involvement in SSc and could serve as predictors of mortality.
\end{abstract}

\section{Introduction}

Systemic sclerosis (SSc) is a chronic autoimmune disease characterized by skin and internal organ involvement [1]. The pathogenesis of the disease is still today partially understood, despite the significant amount of work that has been done in understanding the process leading to vasculopathy, immune dysregulation, tissue inflammation and eventually fibrosis [2]. Among organ involvement the kidney is frequently affected: autopsy studies showed its presence in $>60 \%$ of the cases [3]. In the majority of the cases kidney involvement is subclinical and may have a variable clinical expression, including decrease of renal function, glomerulonephritis, chronic vasculopathy, and a

\footnotetext{
* Corresponding author at: University of Firenze, Department of Experimental and Clinical Medicine, Division of Rheumatology, Via delle Oblate 4, 50141 Firenze, Italy.

E-mail address: cosimobruni85@gmail.com (C. Bruni).

${ }^{1}$ Equal senior authorship.
} 
specific sensitivity for drug toxicity. The most dramatic clinical expression of acute kidney involvement in SSc is the well-known scleroderma renal crisis which, in the majority of the cases, is a lethal complication [4]. In these patients, renal pathology studies showed vascular hyperplasia and fibrinoid necrosis at inter-lobular and arcuate renal arteries, with glomeruli sparing or late ischemic damage in uncontrolled cases [5].

In SSc, kidney function serum and urinary tests are part of the assessment of the patient during the follow-up. In particular, we know today that the serum creatinine measurement is affected by muscle mass reduction and creatinine clearance impairment [6,7] and proteinuria is having a proper prognostic impact [8]. Frequently, SSc patients are also affected by arterial hypertension as a comorbidity, being a pivotal determinant of renal filtration.

Today, ultrasound is among the first-line tools to assess the kidney and the urinary tract [9]. Moreover, the Doppler technique can evaluate arterial and parenchymal vascular perfusion up to smaller inter-lobar and arcuate arteries, with continuous flow and identifiable systolic peak and diastolic curve [10]. Renal Resistive Index (RRI) is a semi-quantitative index representing the resistance that blood flow encounters distally at any point where it is measured, with minimal variations from renal to inter-lobular arteries [11]. RRI is calculated as the difference of peak systolic velocity and peak diastolic velocity, divided by the peak systolic velocity. These values are derived by the mean of three measurements performed at renal artery level with Doppler ultrasound [12].

Initially, 0.70 was proposed as a single pathologic RRI cut-off (being pathologic for $R R I \geq 0.70$ ) in obstructive and nonobstructive dilatation of the renal calix [13]. However, later studies demonstrated the pivotal role of age in determining RRI values and age-adjusted RRI cut-offs were proposed [14]. RRI can be influenced by local renal factors, including both vascular resistance and compliance, but also by non-vascular factors that increase pressure in interstitial compartment and in SSc are mostly due to fibrosis $[15,16]$. Systemic determinants are also well known, such as arterial hypertension [17], arteriosclerosis [18] while low-grade inflammation [19], diabetes mellitus [20], hyperuricaemia [21] are less clear but possible causes of renal vasculopathy or tubular-interstitial nephropathy.

Several studies examined the pattern of RRI in SSc patients. In 1996, increased RRI values was been found to correlate with disease duration [22]. Other studies demonstrated that in SSc RRI significantly correlated with glomerular filtration rate, progressive worsening of nailfold video-capillaroscopy (NVC) pattern [23] and a history of digital ulcers [24]. Moreover, RRI variations over time reflected the clinical evolution of a case of scleroderma renal crisis [25] Further, RRI could detect changes in vascular resistance during vasodilating treatment [26]. Although all these data support the use of RRI to detect renal damage in SSc patients, they were derived from small cohorts and by studies focussed on vascular and renal function disease features only, not exploring relationship with skin and fibrotic features as well as prognostic implication.

The aim of our study was to evaluate the pattern of RRI in SSc patients in a retrospective observational study. We also investigated the utility of RRI measurement in clinical practice by studying its determinants and its correlations with clinical, serological and radiological SSc-related features; finally, we analysed the prognostic role of RRI on organ specific worsening or death in SSc patients.

\section{Materials and methods}

SSc patients, classified with the 2013 ACR/EULAR criteria [27] or diagnosed through the Very Early Diagnosis of Systemic Sclerosis criteria [28], attending the Rheumatology Division AOUC of the University of Florence, were enrolled in the study if the RRI measurement was performed at least once after SSc diagnosis and written informed consent was given. The study was approved by local IRB. Data were retrospectively collected from patients' charts as follows: demographics (age at RRI measurement, gender, height, weight, body mass index), clinical non SSc features (diagnosis of arterial hypertension, hyperuricaemia, diabetes mellitus, hyperlipidaemia, smoking exposure), SSc clinical manifestations (time from Raynaud's phenomenon onset, time from first non-Raynaud's symptom, disease subset according to Leroy and Medsger [1], modified Rodnan skin score -mRSS- [29], VEDOSS criteria fulfilment, current or previous history of digital ulcer (DU) [30], telangiectasia, history/diagnosis of pulmonary arterial hypertension by right heart catheterization (PAH), interstitial lung disease (ILD), scleroderma renal crisis, arthritis, presence of tendon friction rubs, gastro-oesophageal involvement, intestinal involvement, presence of dyspnoea), serological and urinary tests (increase of erythrocyte sedimentation rate (ESR) above local laboratory upper limit of normal, increase of C-reactive protein (CRP) above local laboratory upper limit of normal, 24 hours urine protein, NTproBNP, serum creatinine, creatinine clearance calculated with Cockroft-Gault method (creatinine CG), positivity for antinuclear antibodies-ANA-, anti-topoisomerase I antibodies - ATA-, anti-centromere antibodies -ACA-, anti-RNA polymerase III antibodies -ARA), radiology and instrumental assessments (NVC pattern) [31], ejection fraction, estimated systolic pulmonary arterial pressure (sPAP) and presence of diastolic dysfunction on transthoracic echocardiography, forced vital capacity (FVC), absolute and corrected for alveolar volume lung diffusion for Carbone monoxide (DLCO and DLCO/VA) on pulmonary function tests, presence of interstitial lung disease with ground glass opacities, reticulation or honeycombing on chest high resolution tomography (HRCT), RRI measured with renal artery echo Doppler, as previously indicated [14]. ILD and skin involvement were labelled as "fibrotic manifestations", while digital ulcers, telangiectasias, nailfold capillaroscopy changes and signs of $\mathrm{PAH}$ as "vascular manifestations".

All patients had also a follow-up evaluation through their last available visit or the development of clinical-instrumental worsening, which was defined as one or more of the following outcomes: 1) increase of mRSS $>5$ units compared to the previous evaluation (as Skin worsening); 2) development of a new digital ulcer or worsening of NVC pattern (as Peripheral vascular worsening); 3) decline of FVC > 15\% or FVC $<80 \%$ with new appearance of ILD on HRCT or ILD extent increase indicated by the radiologist (as Pulmonary worsening); 4) new left heart failure onset requiring medical treatment, new diagnosis of $\mathrm{PAH}$ or new detection of significant ventricular arrhythmia on $24 \mathrm{~h}$ electrocardiogram requiring prompt medical treatment (as Cardiac worsening); 5) new scleroderma renal crisis onset or creatinine clearance reduction $<30 \mathrm{ml} / \mathrm{min}$ (as Renal worsening) and 6) death.

Statistical analysis was performed with SPSS 20 (IBM-SPSS Inc., Chicago, IL, USA): association between categorical variables was tested with Chi-square test (or Fisher exact test as appropriate), Spearman's Rho test was used to investigate correlation between non-parametric variables. Student's $t$-test was used to compare continuous variables without compensation for repeated testing. Variables are presented as mean \pm standard deviation or median (inter-quartile range) according to normal or non-normal distribution, tested through Shapiro-Wilk's test. In all cases, significance threshold was set to $p<.05$.

Determinants of RRI values in the SSc population were analysed through linear regression analysis and multivariate analysis with backward stepwise approach (probability for entry: 0.05, for removal 0.10 ). The pathologic values of RRI for newly proposed age-adjusted cut-off for the SSc were created by dividing our study population into age quartiles, and then RRI values of each age-quartile were used to calculate the 75th percentile. This adjusted percentile was considered as the pathologic cut-off for each age group.

Prognostic evaluation was also made for all abovementioned disease-related features and both RRI interpretations (linear value as continuous variable and dichotomic evaluation as pathologic or nonpathologic), to test their impact in predicting organ worsening or death. Kaplan Meyer curve and Cox regression analyses were performed for 
Table 1

Disease features in the study population and their distributions among categories of Age-SSc adjusted pathologic RRI.

\begin{tabular}{|c|c|c|c|c|}
\hline & \multirow[t]{3}{*}{ Study population (380 pts) } & \multicolumn{3}{|c|}{ Age-SSc Pathologic RRI } \\
\hline & & \multicolumn{2}{|c|}{ Pathologic RRI (131 pts) } & \multirow{2}{*}{$\begin{array}{l}\text { P value (vs non-pathologic } \\
\text { RRI) }\end{array}$} \\
\hline & & $\mathrm{n}$ & $\%$ & \\
\hline Male gender, (n, \%) & $43(12.1)$ & 19 & 15.7 & 0.177 \\
\hline Arterial Hypertension (n, \%) & $127(33.4)$ & 53 & 42.4 & 0.003 \\
\hline Diabetes Mellitus (n, \%) & $14(3.7)$ & 6 & 42.9 & 0.392 \\
\hline Hyperuricemia (n, \%) & $27(7.1)$ & 15 & 55.6 & 0.058 \\
\hline Dyslipidemia (n, \%) & $42(11.1)$ & 11 & 26.2 & 0.581 \\
\hline Skin subset: no skin involvement/limited/diffuse (n, \%) & $\begin{array}{l}75(19.7) / 193(50.8) / 112 \\
(29.5)\end{array}$ & \multicolumn{2}{|c|}{$7(5.8) / 66(54.5) / 48(39.7)$} & $<0.001$ \\
\hline mRSS (median, IQR) & $5(0-12)$ & \multicolumn{2}{|c|}{$8(4-16)$} & $<0.001$ \\
\hline Fulfilment of VEDOSS criteria without other SSc features (n, \%) & $57(15.0)$ & 6 & 5.0 & $<0.001$ \\
\hline History/Current Digital Ulcers (n, \%) & $148(38.9)$ & 64 & 52.9 & $<0.001$ \\
\hline $\begin{array}{l}\text { Nailfold Videocapillaroscopy scleroderma pattern (absent/early/active/ } \\
\text { late) - missing data } 28 \text { patients }\end{array}$ & $\begin{array}{l}33(8.7) / 108(26.8) / 110 \\
(28.9) / 107(28.2)\end{array}$ & \multicolumn{2}{|c|}{$\begin{array}{l}7(6.3) / 24(21.6) / 34(30.6) / 46 \\
(41.4)\end{array}$} & 0.013 \\
\hline Pulmonary arterial hypertension (n, \%) & $23(6.1)$ & 9 & 7.6 & 0.489 \\
\hline Telangectasias (n, \%) & $81(21.4)$ & 35 & 48.6 & 0.017 \\
\hline Systolic PAP on Echo (median, IQR) & $27(22-32)$ & \multicolumn{2}{|c|}{$29(24.34)$} & 0.002 \\
\hline Diastolic Dysfunction (E/A < 1) $(\mathrm{n}, \%)$ & $120(31.6)$ & 41 & 41.4 & 0.476 \\
\hline Interstitial Lung Disease (n, \%) & $113(29.7)$ & 46 & 38.3 & 0.016 \\
\hline FVC $(\%$, mean \pm DS $)$ & $101 \pm 21$ & \multicolumn{2}{|c|}{$98 \pm 21$} & 0.023 \\
\hline DLCO $(\%$, mean \pm DS $)$ & $73 \pm 19$ & \multicolumn{2}{|c|}{$68 \pm 19$} & 0.010 \\
\hline Dyspnea NYHA functional class $\geq 2$ (n, \%) & $25(6.6)$ & 48 & 39.7 & 0.130 \\
\hline ESR increase (n, \%) & $103(27.1)$ & 44 & 40.7 & 0.008 \\
\hline CRP increase (n, \%) & $45(11.8)$ & 20 & 18.7 & 0.086 \\
\hline Scleroderma Renal crisis history, (n, \%) & $6(1.6)$ & 4 & 3.3 & 0.088 \\
\hline Anti-Centromere antibody positivity (n, \%) & $195(51.3)$ & 53 & 44.2 & 0.060 \\
\hline Anti-Topoisomerase I antibody positivity (n, \%) & $137(36.0)$ & 56 & 46.7 & 0.006 \\
\hline Anti- RNA polymerase III antibody positivity (n, \%) & $20(5.3)$ & 8 & 7.2 & 0.459 \\
\hline NTproBNP (median, IQR) & $114(50-304)$ & \multicolumn{2}{|c|}{$222(130-361)$} & 0.001 \\
\hline Cockroft-Gault Calculated Creatinine Clearance (median, IQR) & $97.8(80-120)$ & \multicolumn{2}{|c|}{$97(80-124)$} & 0.822 \\
\hline 24-hours urine protein (mg/dl/24 h) & $100(53-125)$ & \multicolumn{2}{|c|}{$105(80-130)$} & 0.035 \\
\hline
\end{tabular}

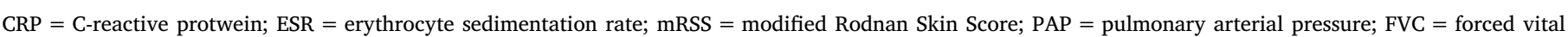

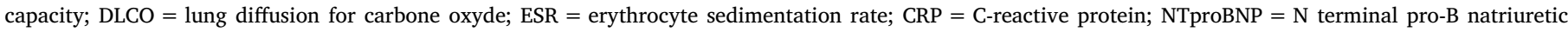
peptide; NYHA = New York Heart Association, RRI = Renal Resistive Index; VEDOSS = very early diagnosis of systemic sclerosis, IQR = interquartile range.

survival analysis and multivariate analysis with backward stepwise approach (probability for entry: 0.05 , for removal 0.10 ) to create a predictive model for pathologic RRI.

The study was approved by Local Ethical Committee (Comitato Etico Regionale per la Sperimentazione Clinica della Regione Toscana, sezione Area Vasta Centro, codice 12350_oss) and patients' signed informed consent was obtained.

\section{Results}

Out of 460 SSc patients evaluated, 380 were considered eligible for the study: 323 fulfilled the 2013 ACR/EULAR SSc criteria and 57 fulfilled the VEDOSS criteria: $12.1 \%$ were male, mean age $55 \pm 15$ years. Median time since Raynaud's phenomenon onset and from first nonRaynaud's symptoms were 9 and 4 years, respectively. A large part out of our SSc population were anti-centromere antibody (195, 51.3\%) positive and presented various clinical features: 305 (81.3\%) had cutaneous fibrotic involvement, 23 (6.1\%) had PAH and $113(29.7 \%)$ had ILD [while $10(2.7 \%)$ presented both]. Further study population characterization is presented in Table 1 .

\subsection{RRI distribution in the SSc population}

RRI values ranged from 0.50 to 0.89 in the study population, mean: $0.68 \pm 0.07$. RRI was positively correlated with age $(\rho=0.564$, $p<.001)$ and negatively correlated with creatinine clearance ( $\rho=-0.321, p<.001$ ), as expected. Similarly, when considering extra-renal determinants of RRI, arterial hypertension $(p<.001)$, hyperuricaemia $(p<.001)$, diabetes mellitus $(p=.001)$ and dyslipidaemia ( $p=.017$ ) were statistically significantly associated with RRI.

Among SSc-related features, only the presence of either limited or diffuse skin fibrosis $(p=.047)$ and late NVC pattern $(p=.006)$ were significantly associated with RRI, while $\operatorname{sPAP}(\rho=0.352, p<.001)$ and DLCO $(\rho=-0.197, p<.001)$ correlated positively and negatively with RRI, respectively. Despite this result, no statistical difference in absolute RRI values was reached in regards to presence/absence of $\mathrm{PAH}, \mathrm{ILD}, \mathrm{DU}$ and no correlation was seen with mRSS values (data not shown).

\subsection{Determinants of RRI in the SSc population}

We decided to investigate which was the impact of general comorbidities and SSc specific features on RRI values (see Table 2).

At univariate regression analysis, all known RRI determinants in general population had an impact on RRI in our SSc population ( $p=.017$ and below), as well as demographic features (age, time from RP onset and time from SSc diagnosis). Among SSc related features, RRI value prediction was seen for many vascular items (SRC history, DU presence/history, PAH diagnosis, sPAP values estimated on Echo), cardiopulmonary parameters (dyspnoea and \%DLCO), presence of lower gastrointestinal involvement and ACA positivity. When combined in the multivariate regression analysis, only age (OR $0.002,95 \% \mathrm{CI}$ $0.002-0.003, p<.001$ ) and sPAP (OR 0.001, 85\% CI 0.000-0.002, $p=.023$ ) were independent predictors of RRI values. 
Table 2

Determinants of Renal Resistive Index in our Systemic Sclerosis population.

\begin{tabular}{|c|c|c|c|c|}
\hline & Univariate linear regression (OR, 95\% CI) & $p$ value & Multivariate linear regression $(\mathrm{OR}, 95 \% \mathrm{CI})$ & $p$ value \\
\hline Age & $0.003(0.002-0.003)$ & $<0.001$ & $0.002(0.002-0.003)$ & $<0.001$ \\
\hline Male gender & $0.005(-0.025-0.027)$ & 0.940 & & \\
\hline Smoke exposure & $-0.001(-0.028-0.025)$ & 0.926 & & \\
\hline Arterial hypertension & $0.059(0.043-0.075)$ & $<0.001$ & $0.014(-0.004-0.032)$ & 0.115 \\
\hline Diabetes Mellitus & $0.079(0.033-0.124)$ & 0.001 & $0.030(-0.003-0.063)$ & 0.073 \\
\hline Hyper-lipidaemia & $0.029(0.005-0.053)$ & 0.017 & $0.001(-0.028-0.030)$ & 0.952 \\
\hline Hyper-uricaemia & $0.049(0.025 .0 .072)$ & $<0.001$ & $0.019(-0.006-0.031)$ & 0.009 \\
\hline Time from Raynaud's Phenomenon onset & $0.002(0.001-0.002)$ & $<0.001$ & $0.000(0.000-0.001)$ & 0.235 \\
\hline Time from disease onset & $0.002(0.001-0.003)$ & 0.001 & $0.000(-0.001-0.001)$ & 0.590 \\
\hline Very early systemic sclerosis (VEDOSS) patients & $0.004(-0.021-0.030)$ & 0.743 & & \\
\hline Cutaneous subset: sine scleroderma / limited / diffuse & $-0.002(-0.016-0.011)$ & 0.729 & & \\
\hline mRSS & $0.000(-0.001-0.001)$ & 0.626 & & \\
\hline Anti-Centromere antibody positivity & $0.014(0.001-0.028)$ & 0.041 & $0.001(-0.013-0.014)$ & 0.920 \\
\hline Anti-Topoisomerase I antibody positivity & $-0.008(-0.022-0.007)$ & 0.304 & & \\
\hline Sclerodermia Renal Crisis history & $0.058(0.003-0.113)$ & 0.040 & $0.038(-0.012-0.089)$ & 0.137 \\
\hline Digital ulcer history/presence & $0.023(0.005-0.040)$ & 0.011 & $0.010(-0.006-0.026)$ & 0.229 \\
\hline Nailfold-videocapillaroscopy pattern & $0.009(0.000-0.018)$ & 0.060 & & \\
\hline Late Nailfold-videocapillaroscopy pattern & $0.014(-0.006-0.033)$ & 0.171 & & \\
\hline Pulmonary Arterial Hypertension & $0.042(0.005-0.079)$ & 0.026 & & \\
\hline Systolic PAP on Echo & $0.002(0.001-0.003)$ & $<0.001$ & $0.001(0.000-0.002)$ & 0.023 \\
\hline Telangectasias & $0.020(-0.003-0.043)$ & 0.089 & & \\
\hline Interstitial Lung Disease & $0.003(-0.017-0.023)$ & 0.758 & & \\
\hline Dyspnea NYHA class $\geq 2$ & $0.024(0.006-0.043)$ & 0.011 & $-0.005(-0.019-0.010)$ & 0.535 \\
\hline$\% \mathrm{FVC}$ & $0.000(0.000-0.000)$ & 0.921 & & \\
\hline$\%$ DLCO & $-0.001(-0.001-0.000)$ & 0.028 & $0.000(-0.001-0.000)$ & 0.411 \\
\hline Upper GI symptoms & $0.004(-0.014-0.023)$ & 0.642 & & \\
\hline Lower GI symptoms & $0.029(0.003-0.055)$ & 0.030 & $0.013(-0.006-0.032)$ & 0.194 \\
\hline Arthritis & $0.021(-0.004-0.046)$ & 0.092 & & \\
\hline Tendon friction rubs & $0.015(-0.018-0.047)$ & 0.376 & & \\
\hline NTproBNP level & $0.000(0.000-0.000)$ & 0.037 & $0.000(0.000-0.000)$ & 0.074 \\
\hline Cockroft-Gault Calculated Creatinine Clearance & $-0.001(-0.001-0.000)$ & $<0.001$ & $0.000(-0.001-0.001)$ & 0.586 \\
\hline 24-hours urine protein level & $0.000(0.000-0.000)$ & 0.002 & $0.000(0.000-0.000)$ & 0.922 \\
\hline
\end{tabular}

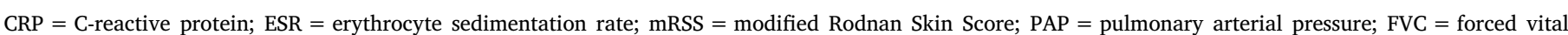

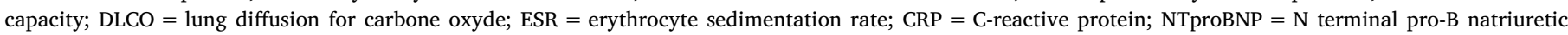
peptide; NYHA = New York Heart Association; RRI = Renal Resistive Index; IQR = interquartile range.

\subsection{Proposed age-SSc adjusted pathologic cut-offs}

As none of the general population comorbidities determinants independently predicted RRI, we decided to create age-SSc adjusted cutoffs, as previously explained (Fig. 1 and Table 3 ).

Hundred-thirty-one $(31.8 \%)$ patients had a pathologic RRI. When testing general population determinants, a significant association was still confirmed with arterial hypertension $(p=.003)$, but not with hyperuricaemia, dyslipidaemia, diabetes mellitus or creatinine clearance ( $\mathrm{p}=\mathrm{NS}$ ). On the other hand, patients with age-SSc adjusted pathologic RRI showed a significantly higher prevalence of fibrotic and vascular manifestations. In particular, the limited/diffuse skin fibrosis $(94.2 \%$ vs $81.3 \%, p<.001$ ) and mRSS (median 8 vs $5, p<.001$ ), history/presence of DU ( $52.9 \%$ vs $38.9 \%, p<.001$ ), sPAP values (median 29 vs $27, p=.002)$, ILD on HRCT ( $38.3 \%$ vs $29.7 \%, p=.016)$ and lower FVC

\section{0 pts}
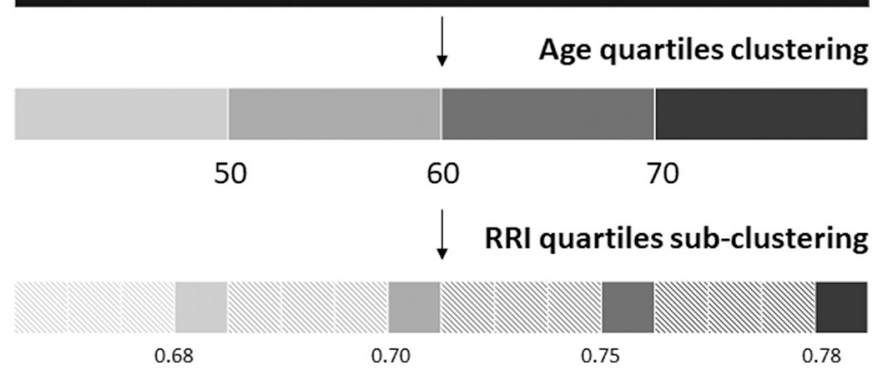

Fig. 1. Methodological algorithm for the determination of Age-SSc Adjusted Renal Resistive Index Pathologic cut-offs
Table 3

Proposed age-SSc adjusted pathologic cut-offs for Renal Resistive Index.

\begin{tabular}{ll}
\hline Age (years) & Age-SSc adjusted Pathologic RRI \\
\hline 1st quartile $\leq 49$ y & $\geq 0.68$ \\
2nd quartile $50-59$ y & $\geq 0.70$ \\
3rd quartile 60-69 y & $\geq 0.75$ \\
4th quartile $\geq 70$ y & $\geq 0.78$ \\
\hline
\end{tabular}

RRI = Renal Resistive Index.

$(98 \pm 21$ vs $101 \pm 21, p=.023)$ and lower DLCO (68 \pm 19 vs $73 \pm 19, p=.010$ ) were significantly more prevalent in the Age-SSc adjusted pathologic population. In line with the higher prevalence of ILD and higher mRSS, Scl70 were also significantly associated with pathologic RRI (46.7\% vs $36.0 \%, p=.006)$. Interestingly, only $10.5 \%$ of VEDOSS patients had a pathologic RRI, significantly lower than those with definite SSc $(10.5 \%$ vs $35.9 \%, p<.001)$.

\subsection{Prognostic value of RRI}

Data for follow-up were available for all patients, with a mean follow up close to 3 years ( $37 \pm 27$ months, median 32 months, range $0.3-128$ months). One-hundred-eighty patients (47.60\%) presented at least one organ system that worsened or death, with peripheral vascular (17.6\%), cardiac (12.6\%) and lung (17.4\%) being the most frequent complications.

Higher baseline absolute RRI value showed statistically significant association with death, cardiac worsening and renal worsening ( $p=.001, p=.017$ and $p=.043$ respectively), while no significant association was found between Age-SSc pathologic RRI and worsening outcomes (see Table 4). 
Table 4

Prevalence of worsening according to different interpretations of RRI values.

\begin{tabular}{|c|c|c|c|c|c|c|c|}
\hline & \multicolumn{4}{|c|}{ Study population (380 pts) } & \multicolumn{3}{|c|}{ Age-SSc corrected pathologic RRI (131 pts) } \\
\hline & $\mathrm{n}$ & $\%$ & Mean RRI $\pm S D$ & $\mathrm{p}$ value & $\mathrm{n}$ & $\%$ & $\mathrm{p}$ value \\
\hline General worsening & 180 & $47.60 \%$ & $0,69 \pm 0,08$ & 0.098 & 37 & $51,39 \%$ & 0,197 \\
\hline Death & 26 & $6.80 \%$ & $0,71 \pm 0,10$ & 0.001 & 12 & $9.90 \%$ & 0.128 \\
\hline Skin worsening & 23 & $6.10 \%$ & $0,68 \pm 0,07$ & 0.875 & 11 & $9.10 \%$ & 0.108 \\
\hline Peripheral vascular worsening & 67 & $17.60 \%$ & $0,69 \pm 0,07$ & 0.367 & 21 & $17.40 \%$ & 0.955 \\
\hline Cardiac worsening & 48 & $12.60 \%$ & $0,71 \pm 0,08$ & 0.017 & 20 & $16.50 \%$ & 0.137 \\
\hline Pulmonary worsening & 66 & $17.4 \%$ & $0,70 \pm 0,08$ & 0.523 & 20 & $16.70 \%$ & 0.775 \\
\hline Renal worsening & 16 & $4.20 \%$ & $0,72 \pm 0,07$ & 0.043 & 7 & $5.80 \%$ & 0.411 \\
\hline
\end{tabular}

RRI $=$ Renal Resistive Index

Table 5

Univariate and multivariate Cox regression analysis for predicting death.

\begin{tabular}{|c|c|c|c|c|}
\hline & Univariate linear regression - OR (95\% CI) & $p$ value & Multivariate linear regression - OR (95\% CI) & $p$ value \\
\hline Age & $1.053(1.017-1.019)$ & 0.001 & $1.138(0.989-1.309)$ & 0.071 \\
\hline Male gender & $1.081(0.682-4.855)$ & 0.231 & & \\
\hline Limited cutaneous subset & $0.898(0.409-1.970)$ & 0.787 & & \\
\hline mRSS & $1.069(1.027-1.111)$ & 0.001 & $1.070(1.007-1.136)$ & 0.028 \\
\hline Anti-Centomere antibody positivity & $0.812(0.370-1.779)$ & 0.602 & & \\
\hline Anti-Topoisomerase I antibody positivity & $1.161(0.521-2.587)$ & 0.715 & & \\
\hline RNApolIII positivity & $0.046(0.000-106.669)$ & 0.435 & & \\
\hline Scleroderma Renal crisis & $4.886(0.655-35.466)$ & 0.122 & & \\
\hline Digital ulcer history/presence & $2.865(1.265-6.489)$ & 0.012 & $1.439(0.422-4.902)$ & 0.189 \\
\hline Late NVC pattern & $4.599(1.855-11.401)$ & 0.001 & $2.841(1.007-8.013)$ & 0.048 \\
\hline Pulmonary Arterial Hypertension & $4.094(1.529-10.958)$ & 0.005 & $1.436(0.364-5.657)$ & 0.200 \\
\hline Systolic PAP on Echo & $1.052(1.027-1.077)$ & $<0.001$ & $1.028(0.984-1.073)$ & 0.212 \\
\hline Left ventricule ejection fraction & $0.975(0.925-1.029)$ & 0.360 & & \\
\hline Telangectasias & $8.910(1.975-40.208)$ & 0.004 & & \\
\hline Interstitial Lung Disease & $6.117(2.613-14.319)$ & $<0.001$ & $4.741(1.848-12.163)$ & 0.001 \\
\hline Dyspnea NYHA class $\geq 2$ & $7.720(3.199-18.632)$ & $<0.001$ & $5.143(1.744-15.143)$ & 0.003 \\
\hline Arthritis & $1.417(0.485-4.144)$ & 0.524 & & \\
\hline Tendon friction rubs & $1.121(0.263-4.761)$ & 0.877 & & \\
\hline Increased CRP & $6.222(2.453-15.786)$ & $<0.001$ & $2.017(0.623-6.384)$ & 0.233 \\
\hline NTproBNP level & $1.001(1.000-1.001)$ & 0.004 & $1.000(0.998-1.003)$ & 0.743 \\
\hline Cockroft-Gault Calculated Creatinine Clearance & $0.980(0.965-0.995)$ & 0.008 & $0.983(0.964-1.001)$ & 0.070 \\
\hline 24-hours urine protein level & $1.004(1.002-1.006)$ & $<0.001$ & $1.002(0.997-1.007)$ & 0.378 \\
\hline RRI (for 0.01 increase) & $2.210(1.892-170.210)$ & $<0.001$ & $4.816(1.002-2505)$ & 0.048 \\
\hline Age-SSc adjusted pathologic RRI & $2.266(1.032-4.976)$ & 0.041 & $1.687(0.188-2.518)$ & 0.571 \\
\hline
\end{tabular}

$\mathrm{CRP}=\mathrm{C}$-reactive protein; ESR = erythrocyte sedimentation rate; mRSS = modified Rodnan Skin Score; PAP = pulmonary arterial pressure; ESR $=$ erythrocyte sedimentation rate; $\mathrm{CRP}=\mathrm{C}$-reactive protein; NTproBNP $=\mathrm{N}$ terminal pro-B natriuretic peptide; NYHA = New York Heart Association; RRI = Renal Resistive Index; $\mathrm{IQR}=$ interquartile range, $\mathrm{NVC}=$ Nailfold videocapillaroscopy.
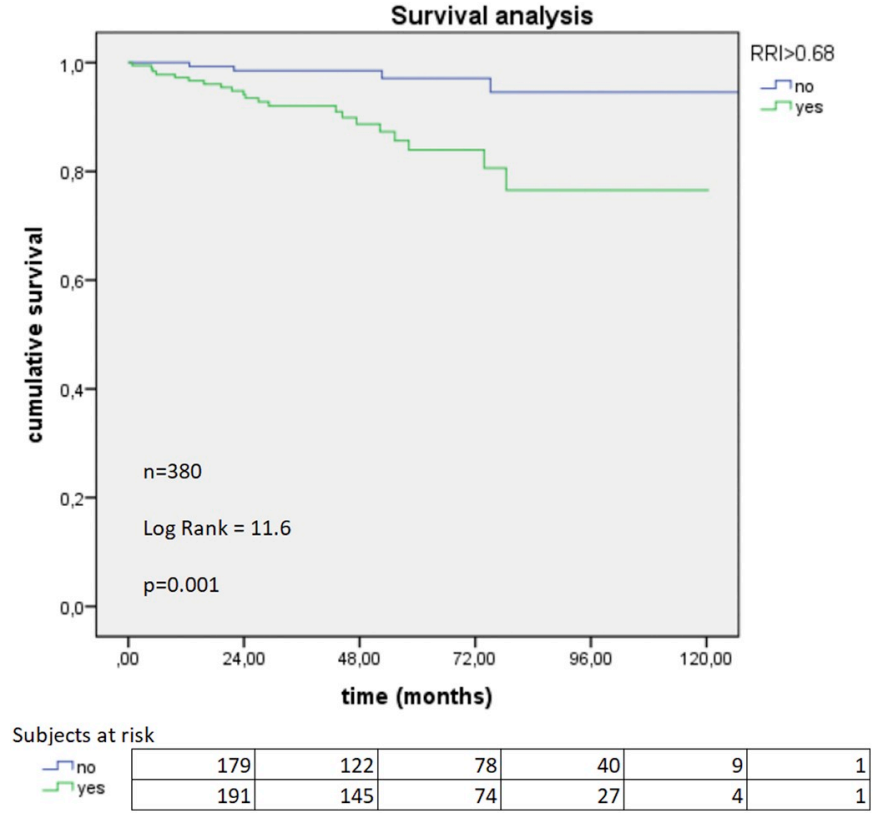

Fig. 2. Kaplan-Meier curves and estimates of event-free survival in patients with $>=$ or $<0.68$ renal resistive index. (event $=$ death)
When survival analysis was performed to test the prognostic impact of RRI in our SSc population, univariate regression analysis showed statistical significance for both absolute RRI value and Age-SSc adjusted pathologic RRI in predicting death (HR 2.210 CI95\% 1.892-170.210 $p<.001$ and HR 2.266 CI95\% 1.032-4.976 $p=.041$ ), while only for absolute RRI value in predicting cardiac worsening (HR $4.961 \mathrm{CI} 95 \%$ $1.678-527.815 p=.009$ ) and renal worsening (HR $1.432 \mathrm{CI} 95 \%$ $1.102-661.761 p=.026)$.

Absolute RRI value was confirmed as an independent predictor of mortality (HR 4.816, CI95\% 1.002-2505, $p=.048$ ), together with mRSS, late NVC pattern, presence of ILD and dyspnoea with NYHA functional class $\geq 2$ (see Table 5 ).

A ROC curve analysis identified RRI value of 0.68 as the best cut-off to predict mortality (AUC $=0.696,95 \%$ CI $0.591-0.801, p=.001$ ), with $88.5 \%$ sensitivity and $50.9 \%$ specificity (Fig. 1 shows KaplanMeier survival analysis). For cardiac worsening, male gender was the strongest independent predictor, together with mRSS and NTproBNP levels (Online Supplement 1) (Fig. 2).

\section{Discussion}

Our data show that, in this retrospective hypothesis generating study, age-SSc adjusted pathologic RRI appears to be a potential marker 
for fibrosis and vasculopathy in SSc. Moreover, increased absolute and age-SSc adjusted RRI values seems to predict mortality.

In arterial hypertension, diabetes mellitus and hyperuricaemia, RRI is a useful marker in detecting early renal damage [12]. Conversely in SSc the daily utility of RRI in clinical practice has not been confirmed so far, although various population studies with small-medium sample size reported associations with clinical and instrumental SSc related manifestations. Since age-independent fixed or age-adjusted cut-offs have been previously proposed for the general population, our study shows that age-SSc adjusted pathologic cut-offs are necessary when evaluating SSc patients. This was particularly true for our study population, which is relatively young (55 \pm 15 years). This may, in fact, underestimate the pathology, by using a cut-off determined from an older population [13].

Our study, in the univariate cross-sectional analysis, confirms the previously reported associations between RRI and renal function indexes, arterial hypertension, diabetes mellitus, hyperlipidaemia and hyperuricaemia.

These factors are, in fact, known determinants in increasing vascular stiffness and altering tubular-interstitial renal component that affect RRI value [12]. In particular, the significantly reduced creatinine clearance in the subgroup of patients with pathologic high RRI is in line with previous results, although the whole study population presented on average a well-preserved renal function $\left(97.8 \mathrm{ml} / \mathrm{min} / 1,73 \mathrm{~m}^{2}\right)$ [32]. These preliminary results, supporting the role of RRI as an early renal damage marker, make RRI measurement feasible and applicable also to SSc population. In particular, this may be true when SSc is associated with known cardio-vascular risk factors (arterial hypertension, dyslipidaemia).

In other SSc populations, higher RRI values were detected in patients with a more advanced NVC pattern, and the presence of DU and $\mathrm{PAH}[23,33]$. Although interesting, no pathologic cut-off was applied in these small cohorts, nor for prognostication. Our study included a much larger population of 380 SSc patients, with a wide age and clinical manifestation range, covering almost all disease-related features.

Our analysis following the creation of age-adjusted cut-offs for the SSc population was based on the fact that we found an average RRI value $(0.69 \pm 0.07)$ which is very close to the 0.70 general population pathologic cut-off. When patients were divided into age groups, values were still significantly higher than the average RRI for the same age of non-SSc population [14]. As previously reported, these increased values were not related to time from Raynaud's phenomenon onset or disease duration [34]. In fact, the initial statistically significant correlation of disease duration and pathologic RRI was not confirmed when age was considered. In our cases, we thought that the increased RRI value could be due to initial interstitial renal fibrosis, present from the earliest disease phase and mostly not clinically evident in later evaluations [35]. Autopsy studies reported that fibrotic tubular-interstitial component can be associated with vascular hyperplasia, particularly evident in case of chronic damage due to scleroderma renal crisis [4].

All the background results led us to create age-SSc adjusted pathologic cut-offs which turned out to be relatively close to the general population pathologic value of 0.70 . This cut off was also increased with age, leading to the exclusion of pure age-determined increased RRI values from being considered pathologic. This new cut-off was not significantly associated with impaired renal function (creatinine clearance), while significant association was found with fibrotic, cardiac and vasculopathic SSc manifestations. In particular, the association between Age-SSc adjusted pathologic RRI and lung involvement was confirmed both with chest HRCT and functional assessment (FVC and DLCO). Statistical significance was reached both for limited and diffuse SSc vs early and sine scleroderma SSc, and for the mRSS quantification of skin thickening. These two observations support Age-SSc adjusted pathologic RRI as a disease-related fibrosis biomarker.

Regarding the vascular involvement, previous associations were confirmed [23] both for advanced microvascular changes on NVC and for the presence/history of DU. For the first time, a very strong trend to significant association with the presence of telangiectasias $(p=.017)$, a manifestation of the microcirculatory involvement, was also shown.

From the immunological point of view, Age-SSc adjusted pathologic RRI was associated with ATA positivity, while ACA positivity showed a trend as protective factor. This result might be expected as the two specific autoantibodies are usually mutually exclusive: ATA is a typical marker of diffuse skin involvement with high risk for ILD while ACA was previously shown as a risk factor for DU [36], but not associated with pathologic RRI, hypothesising a protective role.

In our study, VEDOSS patients represented $15.0 \%$ of the population and their absolute RRI values were similar to the remaining SSc population. On the other hand, the Age-SSc adjusted pathologic cut-off was present in few VEDOSS patients $(10.5 \%$ vs $35.9 \%, p<.001)$, being associated with a lower incidence of worsening.

Our data in SSc patients focus the attention on the prognostic role of RRI in the disease. Despite not being an independent predictor of specific organ worsening, RRI values were an independent risk factor for death, together with mRSS (indirectly representing diffuse cutaneous subset), late NVC pattern, presence of ILD and dyspnoea with NYHA functional class $\geq 2$. These results are partially in line with the EUSTAR cohort data where, in a large cohort, the authors identified multiple predictive factors and proposed a prognostic algorithm [37]. We identified RRI and late NVC pattern, not included in the previous EUSTAR analysis, as risk factors for death by all causes in our population. It is well known that NVC alterations are a marker of the underlying pathogenetic vasculopathic processes [2] and that they are associated with vascular disease manifestations, such as DU, PAH and SRC [31]. Compared to NVC, RRI represents either the fibrotic and the vasculopathic SSc burden, as we have shown that it was associated with specific disease features, and could, therefore, be representative for all possible disease manifestations and for their impact on SSc patients' mortality.

\section{Conclusion}

Our study, performed on a large SSc population, proposes RRI as a marker for vascular and in particular fibrotic manifestation in the evaluation of both renal and extra-renal involvement. This result is very close to what is already done in practice with DLCO and SPAP, which are employed for the initial evaluation of the parenchymal and vascular lung involvement, respectively. At any time, a pathological RRI value suggests already a renal, fibrotic and vascular involvement, requiring further assessments. Consequently, given the negative impact that it has on mortality, it should be taken into consideration together with the other known disease specific manifestations and assessments. Further studies in SSc on the role of renal Doppler ultrasound, in particular concerning the sensitivity to change and responsiveness to treatment, are warranted.

\section{Declaration of Competing Interest}

No conflict of interest declared.

\section{Appendix A. Supplementary data}

Supplementary data to this article can be found online at https:// doi.org/10.1016/j.ejim.2019.09.001.

\section{References}

[1] LeRoy EC, Black C, Fleischmajer R, Jablonska S, Krieg T, Medsger Jr. TA, et al. Scleroderma (systemic sclerosis): classification, subsets and pathogenesis. J Rheumatol 1988;15(2):202-5. [Epub 1988/02/01. PubMed PMID: 3361530].

[2] Varga J, Trojanowska M, Kuwana M. Pathogenesis of systemic sclerosis: recent insights of molecular and cellular mechanisms and therapeutic opportunities. J scleroderma rel disord 2017;2(3):137-52. 
[3] Trostle DC, Bedetti CD, Steen VD, Al-Sabbagh MR, Zee B, Medsger Jr. TA. Renal vascular histology and morphometry in systemic sclerosis. A case-control autops study. Arthritis Rheum 1988;31(3):393-400. [Epub 1988/03/01. PubMed PMID: 3358801].

[4] Shanmugam VK, Steen VD. Renal manifestations in scleroderma: evidence for subclinical renal disease as a marker of vasculopathy. Int J Rheumatol 2010;2010. https://doi.org/10.1155/2010/538589. Epub 2010/09/10. (PubMed PMID: 20827302; PubMed Central PMCID: PMCPMC2933853).

[5] Bruni C, Cuomo G, Rossi FW, Praino E, Bellando-Randone S. Kidney involvement in systemic sclerosis: from pathogenesis to treatment. J Scleroderma Rel Disord 2018;3(1):43-52.

[6] Kingdon EJ, Knight CJ, Dustan K, Irwin AG, Thomas M, Powis SH, et al. Calculated glomerular filtration rate is a useful screening tool to identify scleroderma patients with renal impairment. Rheumatology (Oxford) 2003;42(1):26-33. Epub 2003/01/ 02. [PubMed PMID: 12509609].

[7] Scheja A, Bartosik I, Wuttge DM, Hesselstrand R. Renal function is mostly preserved in patients with systemic sclerosis. Scand J Rheumatol 2009;38(4):295-8. Epub 2009/02/14 https://doi.org/10.1080/03009740802629424. [PubMed PMID: 19214870].

[8] Seiberlich B, Hunzelmann N, Krieg T, Weber M, Schulze-Lohoff E. Intermediate molecular weight proteinuria and albuminuria identify scleroderma patients with increased morbidity. Clin Nephrol 2008;70(2):110-7. [Epub 2008/09/17. PubMed PMID: 18793526].

[9] Grenier N, Trillaud H. Comparison of imaging methods for renal artery stenosis. BJU Int 2000;86(Suppl. 1):84-94. [Epub 2000/08/29. PubMed PMID: 10961279].

[10] Rabbia CDLR, Cirillo R. Eco-color-Doppler vascolare. I vasi renali. 2nd ed.Minerva medica; 1995.

[11] Knapp R, Plotzeneder A, Frauscher F, Helweg G, Judmaier W, zur Nedden D, et al. Variability of Doppler parameters in the healthy kidney: an anatomic-physiologic correlation. J Ultrasound Med 1995;14(6):427-9. [Epub 1995/06/01. PubMed PMID: 7658509].

[12] Boddi M, Natucci F, Ciani E. The internist and the renal resistive index: truths and doubts. Intern Emerg Med 2015;10(8):893-905. Epub 2015/09/05 https://doi.org/ 10.1007/s11739-015-1289-2. [PubMed PMID: 26337967].

[13] Platt JF, Rubin JM, Ellis JH. Distinction between obstructive and nonobstructive pyelocaliectasis with duplex Doppler sonography. AJR Am J Roentgenol 1989;153(5):997-1000. Epub 1989/11/01 https://doi.org/10.2214/ajr.153.5.997. [PubMed PMID: 2679004].

[14] Boddi M, Sacchi S, Lammel RM, Mohseni R, Serneri GG. Age-related and vasomotor stimuli-induced changes in renal vascular resistance detected by Doppler ultrasound. Am J Hypertens 1996;9(5):461-6. [Epub 1996/05/01. PubMed PMID: 8735177].

[15] Mostbeck GH, Kain R, Mallek R, Derfler K, Walter R, Havelec L, et al. Duplex Doppler sonography in renal parenchymal disease. Histopathologic correlation. J Ultrasound Med 1991;10(4):189-94. [Epub 1991/04/01. PubMed PMID: 2051529].

[16] Murphy ME, Tublin ME. Understanding the Doppler RI: impact of renal arterial distensibility on the RI in a hydronephrotic ex vivo rabbit kidney model. $J$ Ultrasound Med 2000;19(5):303-14. [Epub 2000/05/16. PubMed PMID 10811403].

[17] Pontremoli R, Viazzi F, Martinoli C, Ravera M, Nicolella C, Berruti V, et al. Increased renal resistive index in patients with essential hypertension: a marker of target organ damage. Nephrol Dial Transplant 1999;14(2):360-5. [Epub 1999/03/ 09. PubMed PMID: 10069189].

[18] Safar ME, London GM, Plante GE. Arterial stiffness and kidney function. Hypertension. 2004;43(2):163-8. Epub 2004/01/21 https://doi.org/10.1161/01. HYP.0000114571.75762.b0. [PubMed PMID: 14732732].

[19] Johnson RJ, Herrera-Acosta J, Schreiner GF, Rodriguez-Iturbe B. Subtle acquired renal injury as a mechanism of salt-sensitive hypertension. $\mathrm{N}$ Engl $\mathrm{J}$ Med 2002;346(12):913-23. Epub 2002/03/22 https://doi.org/10.1056/ NEJMra011078. [PubMed PMID: 11907292].

[20] Ohta Y, Fujii K, Arima H, Matsumura K, Tsuchihashi T, Tokumoto M, et al. Increased renal resistive index in atherosclerosis and diabetic nephropathy assessed by Doppler sonography. J Hypertens 2005;23(10):1905-11. [Epub 2005/09/09. PubMed PMID: 16148615].

[21] Boddi M, Cecioni I, Poggesi L, Fiorentino F, Olianti K, Berardino S, et al. Renal resistive index early detects chronic tubulointerstitial nephropathy in normo- and hypertensive patients. Am J Nephrol 2006;26(1):16-21. Epub 2006/01/13 https:// doi.org/10.1159/000090786. [PubMed PMID: 16401882].

[22] Rivolta R, Mascagni B, Berruti V, Quarto Di Palo F, Elli A, Scorza R, et al. Rena vascular damage in systemic sclerosis patients without clinical evidence of nephropathy. Arthritis Rheum 1996;39(6):1030-4. [Epub 1996/06/01. PubMed PMID: 8651967].

[23] Rosato E, Gigante A, Barbano B, Cianci R, Molinaro I, Rossi C, et al. Intrarenal hemodynamic parameters correlate with glomerular filtration rate and digital microvascular damage in patients with systemic sclerosis. Semin Arthritis Rheum 2012;41(6):815-21. Epub 2011/12/24 https://doi.org/10.1016/j.semarthrit.2011. 11.005. [PubMed PMID: 22192932].

[24] Gigante A, Barbano B, Granata G, Quarta S, Amoroso A, Salsano F, et al. Evaluation of estimated glomerular filtration rate and clinical variables in systemic sclerosis patients. Clin Nephrol 2016;85(6):326-31. Epub 2016/04/30 https://doi.org/10. 5414/CN108580. [PubMed PMID: 27125627].

[25] Rosato E, Gigante A, Barbano B, Molinaro I, Cianci R, Salsano F. Doppler indices of intrarenal arterial stiffness are useful in monitoring scleroderma renal crisis. Scand J Rheumatol 2013;42(1):80-1. Epub 2012/11/07 https://doi.org/10.3109/ 03009742.2012.723748. [PubMed PMID: 23126530].

[26] Scorza R, Rivolta R, Mascagni B, Berruti V, Bazzi S, Castagnone D, et al. Effect of iloprost infusion on the resistance index of renal vessels of patients with systemic sclerosis. J Rheumatol 1997;24(10):1944-8. [Epub 1997/10/23. PubMed PMID: 9330936].

[27] van den Hoogen F, Khanna D, Fransen J, Johnson SR, Baron M, Tyndall A, et al 2013 classification criteria for systemic sclerosis: an American College of Rheumatology/European League against Rheumatism collaborative initiative. Arthritis Rheum 2013;65(11):2737-47. Epub 2013/10/15 https://doi.org/10. 1002/art.38098. [PubMed PMID: 24122180; PubMed Central PMCID: PMCPMC3930146]

[28] Avouac J, Fransen J, Walker UA, Riccieri V, Smith V, Muller C, et al. Preliminary criteria for the very early diagnosis of systemic sclerosis: results of a Delphi consensus study from EULAR scleroderma trials and research group. Ann Rheum Dis 2011;70(3):476-81. Epub 2010/11/18 https://doi.org/10.1136/ard.2010.136929. [PubMed PMID: 21081523].

[29] Khanna DFDE, Clements PJ, et al. Standardization of the modified Rodnan skin score for use in clinical trials of systemic sclerosis. J scleroderma relat disord 2017;2(1):11-8. https://doi.org/10.5301/jsrd.5000231.

[30] Suliman YABC, Johnson SR, Praino E, Aleman H, Borazan N, Cometi L, et al. Defining skin ulcers in systemic sclerosis: systematic literature review and proposed World Scleroderma Foundation (WSF) definition. J scleroderma relat disord 2017;2(2):115-20.

[31] Ruaro B, Sulli A, Smith V, Pizzorni C, Paolino S, Alessandri E, et al. Advances in nailfold capillaroscopic analysis in systemic sclerosis. J Scleroderma Rel Disord 2018;3(2):122-31. https://doi.org/10.1177/2397198318757699.

[32] Bruno RM, Daghini E, Landini L, Versari D, Salvati A, Santini E, et al. Dynamic evaluation of renal resistive index in normoalbuminuric patients with newly diagnosed hypertension or type 2 diabetes. Diabetologia. 2011;54(9):2430-9. Epub 2011/04/19 https://doi.org/10.1007/s00125-011-2148-y. [PubMed PMID: 21499674].

[33] Gigante A, Barbano B, Barilaro G, Quarta S, Gasperini ML, Di Mario F, et al. Serum uric acid as a marker of microvascular damage in systemic sclerosis patients. Microvasc Res 2016;106:39-43. Epub 2016/03/24 https://doi.org/10.1016/j.mvr. 2016.03.007. [PubMed PMID: 27003713].

[34] Aikimbaev KS, Canataroglu A, Ozbek S, Usal A. Renal vascular resistance in progressive systemic sclerosis: evaluation with duplex Doppler ultrasound. Angiology. 2001;52(10):697-701. Epub 2001/10/23 https://doi.org/10.1177/ 000331970105201006. [PubMed PMID: 11666134].

[35] Steen VD. Scleroderma renal crisis. Rheum Dis Clin North Am 2003;29(2):315-33. Epub 2003/07/05. [PubMed PMID: 12841297].

[36] Denton CP, Krieg T, Guillevin L, Schwierin B, Rosenberg D, Silkey M, et al. Demographic, clinical and antibody characteristics of patients with digital ulcers in systemic sclerosis: data from the DUO registry. Ann Rheum Dis 2012;71(5):718-21. Epub 2012/01/17 https://doi.org/10.1136/annrheumdis-2011-200631. [PubMed PMID: 22247218; PubMed Central PMCID: PMCPMC3329234].

[37] Elhai M, Meune C, Boubaya M, Avouac J, Hachulla E, Balbir-Gurman A, et al Mapping and predicting mortality from systemic sclerosis. Ann Rheum Dis 2017;76(11):1897-905. Epub 2017/08/25 https://doi.org/10.1136/annrheumdis2017-211448. [PubMed PMID: 28835464]. 